\title{
Anesthetic Management of Surgical Repair of Descending Thoracic Aortic Aneurysm
}

\author{
Nabil A Mageed*, Ibrahim I Abd El Baser and Hani I Taman \\ Department of Anesthesia and Surgical Intensive Care, Mansoura University, Egypt
}

*Corresponding author: Nabil A Mageed, Department of Anesthesia and Surgical

Received Date: March 20, 2020

Intensive Care, Mansoura University, Egypt

Published Date: April 06, 2020

\begin{abstract}
Anesthetic management of surgical repair of descending thoracic aortic aneurysm is a difficult mission for anesthesiologists. The surgical approach is best achieved through left postero-lateral thoracotomy with the use of one lung ventilation. The post-operative outcome is affected by the presence of associated co morbidities such as cardiac and renal diseases, the severe intra-operative hemodynamic fluctuations and the use of mechanical circulatory assisted devices. The severe hemodynamic fluctuations that result from clamping and declamping the descending thoracic aorta require an experienced cardiac anesthesiologist for the proper perioperative management of these patients. The aim of this review is to give new insights on the anesthetic management and the techniques of distal perfusion using partial cardiopulmonary bypass. Anesthetic goals are directed to provide spinal cord and renal protection with controlling the proximal hypertension.
\end{abstract}

Keywords: Aorta; Anesthetic management; Thoracic aneurysm; Cardiopulmonary bypass surgery

\section{Introduction}

Descending thoracic aortic aneurysm (DTA) affects different segments of the thoracic aorta and sometimes continues to involve the abdominal part of aorta [1]. Although its incidence is lower than abdominal aortic aneurysms, it represents about $40 \%$ of all thoracic aortic aneurysm cases [1]. This lesion affects mainly male at the age of 59-69 years [2]. The commonest cause of death from DTA is spontaneous or traumatic rupture of the aneurysm. Patients who remain alive after surgical repair of ruptured aneurysm usually suffer from poor postoperative outcome with prolonged hospital length of stay and poor life quality. The operative mortality after surgery on thoracic aorta aneurysm is (5-12) \% with (70-79) \% overall 5 years survival [3].

Anesthetic management of the surgical repair of descending thoracic aortic aneurysm is a difficult and critical mission for anesthesiologists as surgical intervention affects every single organ system in the body, causing multiple comorbidities. Management of such patients requires a full review of the disease pathophysiology, surgical techniques and protective organ strategies [4].

\section{Etiology and Pathophysiology}

Thoracic aorta may be affected by either aneurysm or dissection, occurring separately or together, and may be congenital or acquired. An acquired disease is a result of hypertension and atherosclerosis. Congenital causes include connective tissue diseases (Marfan syndrome, Ehlerse Danlos syndromes and Turner's syndrome) and polycystic kidney disease [5]. A true aortic aneurysm is a permanent dilatation at least $50 \%$ greater than its original size involving all wall layers. A pseudo-aneurysm is caused by rupture of the layers of aortic wall that held together by blood and surrounding tissues. A dissection is a disruption of the intima of the aorta that cause bleeding within the aortic wall resulting in its dissection [5].

\section{Classification of aortic aneurysms}

Thoracoabdominal aortic aneurysms (TAAAs) are described using the Crawford classification (Figure 1) according to the segment involved [6]: 

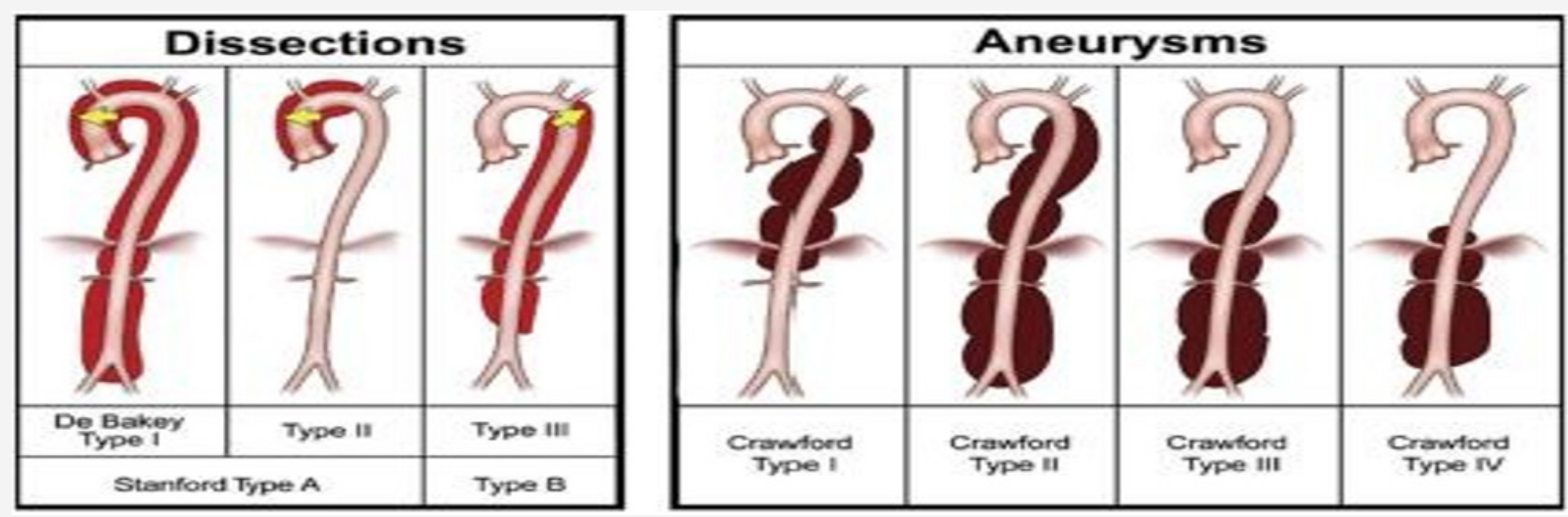

Figure 1: Classification of Aortic Dissection and Aneurysm.

1) Extent I: involves the segment of aorta between the left subclavian artery and the diaphragmatic wall.

2) Extent II: involves the segment of aorta between the left subclavian artery and bifurcation of aorta.

3) Extent III: from the lower half of the descending thoracic aorta to bifurcation of aorta Extent IV: disease confined to the abdominal aorta.

\section{Classification of dissection}

Aortic dissection can be described using DeBakey and Stanford classifications (Figure 1).

1) DeBakey classification

a. Type I: the tear in the intima affects the ascending aorta; involves all portions of the thoracic aorta.

b. Type II: the tear in the intima affects the ascending aorta; involves only the ascending aorta, stopping before the innominate artery.

c. Type III: intimal tear site is in the descending segment; starting just after the origin of the left subclavian artery; can propagate proximally into the arch.

\section{2) Stanford classification}

a. Type A: with any affection of the ascending aorta, intimal tear or extension of dissection may involve any part of ascending aorta; runs a more virulent course.

b. Type B: involves the aortic segment just after the origin the left subclavian artery; generally medically managed except if there is any evidence of a life-threatening complication [7].

Preoperative assessment: Patients presenting with aortic disease often have multiple significant comorbidities. Preoperative assessment, as time allows, should include the following:

a) Assessment of functional capacity and reserve of each organ system for risk stratification and prediction of postoperative complications. Any history of previous cardiac, respiratory, renal or hepatic or neurological disease should be elucidated; Coronary angiography is essential before the procedure. Evaluation of the pulmonary reserve by pulmonary function tests to exclude any contraindication to one lung ventilation (OLV) which is used to facilitate the surgical exposure [8]. Kidney should properly evaluated as the coexisting hypertension and diabetic nephropathy may result in severe postoperative renal dysfunction [8].

b) Examination for evidence of compression of adjacent structures. Stridor or dyspnea indicates encroachment on the trachea or left main bronchus; dysphagia indicates esophageal compression; hoarseness is caused by stretching of the recurrent laryngeal nerve.

c) Baseline neurological examination and recording of any neurological deficit [9].

\section{Diagnostic Imaging}

Three dimensional computed tomographic angiography (CTA) is the gold standard radiologic investigation for diagnosis of aortic aneurysm [10]. Transoesophageal echocardiography (TOE) gives essential information to guide the surgical approach and to anticipate potential problems during the surgery and may be an alternative to CTA if there is no enough time to do before urgent surgery [11].

Surgery involving the descending thoracic aorta: The surgical approach is dependent on the extent of the segment involved with the aneurysm, and the coordination between the anesthesia and surgery teams. Repair of DTA is associated with application of aortic cross-clamp. When a clamp is applied distal to the left subclavian artery, the use of proximal and distal aortic perfusion methods is preferable both to alleviate the raised left ventricular afterload and to maintain perfusion to all body organs [9].

Induction and maintenance of anesthesia: The patient should receive sedative premedication in the preoperative area with reducing the dose of sedatives as the patients are vulnerable to cardiorespiratory depression. In case of dissecting aneurism, the 
surgery will be urgent, a nasogastric tube should be inserted with administration of non-particulate anti acids with performing rapid sequence induction.

In the operative theatre, the patient is attached to five lead electrocardiography (ECG) and pulse oximetry. Right radial artery is cannulated for the pre ductal invasive arterial pressure monitoring, whereas the left radial artery should not be used as the aortic clamp is usually affect the left subclavian artery [12]. Simultaneous right radial and femoral arterial invasive pressure monitoring may help in maintaining the pressure distal to aortic cross clamp during performing partial cardiopulmonary bypass and in monitoring the pressure gradient across the repair to evaluate the adequacy of surgical repair. Anesthesia is induced with propofol $(2 \mathrm{mg} / \mathrm{kg})$ or ketamine (1-2 mg/kg), fentanyl (2-5 $\mu \mathrm{g} / \mathrm{kg}$ ) and rocuronium (1 $\mathrm{mg} / \mathrm{kg}$ ). Anesthesia is maintained with either with isoflurane or sevoflurane with continuous infusion of fentanyl and midazolam [12].

\section{Hemodynamic monitoring and management}

Inserting a large-bore intravenous cannula (16-14F) in a chosen large vein is advised. A central venous catheter should be inserted in the right internal jugular vein which can be replaced by a pulmonary artery catheter after induction of anesthesia to monitor the cardiac filling pressures. The sheath of pulmonary artery catheter is used for central route of drug administration and rapid fluid infusion. Rapid infusion systems with blood- warming capacity are necessary with available six to ten units of cross matched blood, plasma and platelets [13]. Centrifugal device that scavenges erythrocytes should be used [12].

\section{Additional Monitoring:}

1. Transoesophageal echocardiography: TEE can provide instantaneous views of the aorta and confirm exact vessel lesion (Fig 2). It also can accurately confirmed previous angiographic studies delineating aortic pathology, and measurement of aortic dimensions. It allows to visualize the pathology in the distal aortic arch as well as in the distal descending thoracic aorta. Large intercostal arteries can also be imaged. After aneurysm repair, monitoring of blood flow through the aorta can usually be easily confirmed using color-Doppler flow imaging [14]. Lastly, since high percentage of patients suffer from severe associated cardiac disease, TEE allowed dynamic evaluation of cardiac function and volume status. In contrast to angiography, both leakage as well as dissections may easily be diagnosed by TEE [15] (Figure 2) [15].
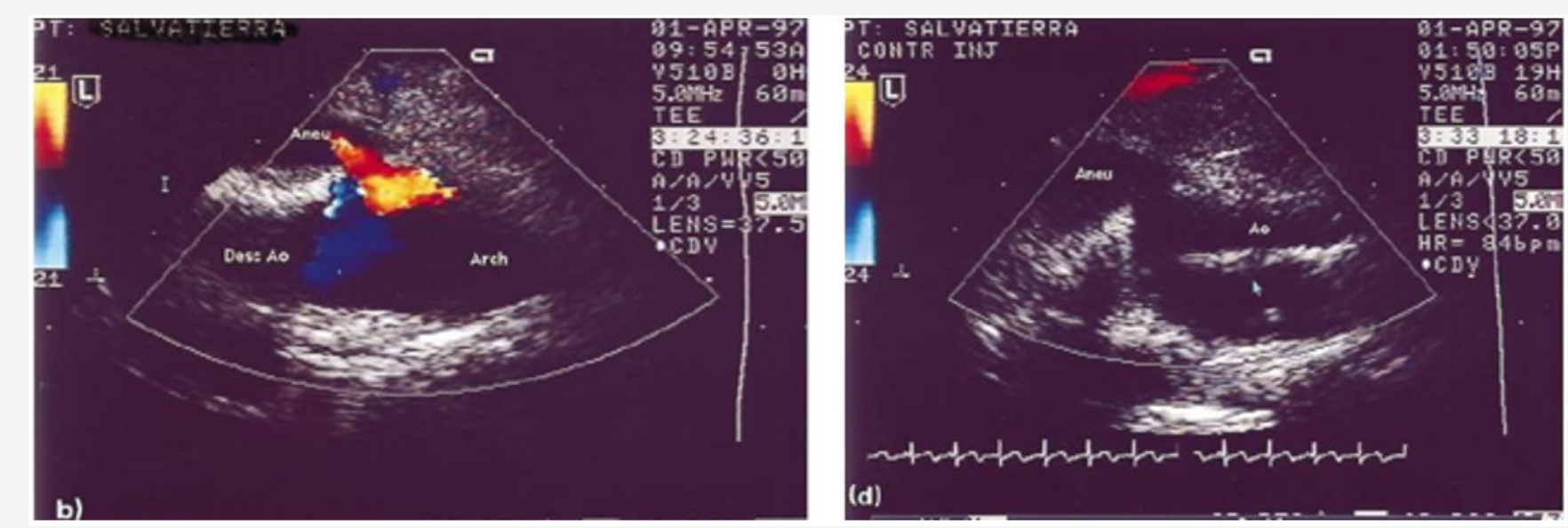

Figure 2: TEE longitudinal axis of the aortic arch and proximal descending thoracic aorta showing DTA aneurysm.

2. Temperature monitoring: Body temperature should be monitored via nasopharynex as it is closely related to cerebral temperature.

3. Transcutaneous near-infrared spectroscopy: NIRS is currently used for cerebral oximetry in cardiovascular surgery. It used to monitor cerebral blood flow non-invasively during the surgery [16].

\section{One-Lung Ventilation}

The surgical approach to the thoracic aortic aneurism is via left postero-lateral thoracotomy. A proper sized, (37 to 41) Fr, left double-lumen endotracheal tube (DLT) is usually used for one lung ventilation and separation. This technique will isolate both lungs and protect the right lung in case of an intrapulmonary hemorrhage or aneurysm rupture into the left main bronchus. A right-sided DLT is only inserted if there is severe distortion of the left main bronchus by the large aneurysms. The use of OLV will facilitate the surgical exposure, avoids the unnecessary iatrogenic lung compression and retraction and isolate the right lung from spread of blood or secretions from the left lung [12].

\section{Positioning}

The patient should be positioned in the cork-screw right lateral decubitus with the body proximal to hip joint at $60^{\circ}$ and distal to hip joint at $30^{\circ}$ from the horizontal. This facilitates the surgical exposure of femoral vessels in the groin if it is needed. An axillary roll is placed to avoid brachial plexus injury, with padding all pressure areas. The left arm is freely positioned in a swimming stroke position. It is properly maintained in such position by applying arm boards or slings [17]. 


\section{Hemodynamic Fluctuations of Cross Clamping and Declamping of Aorta}

Cross-clamping of aorta is associated with severe hemodynamic changes. Afterload abruptly increase with subsequent elevation of blood pressure proximal to the clamp. The preload is also increased secondary to fluid redistribution from veins distal to the clamped segment of aorta. Myocardial ischemia may occur as a result of increase oxygen demand that is caused by increased afterload, preload and contractility [18].

The more proximal application of aortic cross clamp, the more the severe hemodynamic changes. Calcium channel blockers, nitroglycerine, phentolamine, nitroprusside and inhaled volatile anesthetics with prominent vasodilator effects may be used to control the high proximal arterial pressure. Esmolol infusion may protect against myocardial ischemia by reducing afterload and myocardial contractility.

Removal of aortic cross clamp after repairing the aneurysm is usually associated with arterial hypotension due the sudden decrease in afterload, release of vasodilator metabolites, myocardial ischemia and hypovolemia. Hypotension is treated with volume replacement therapy and vasoactive drugs such as phenylephrine and nor adrenaline to maintain mean arterial pressure (80-100) $\mathrm{mm} / \mathrm{Hg}$

\section{The Techniques of Body Perfusion Distal to the Aortic Clamp}

The goals of distal perfusion techniques are to provide adequate tissue perfusion distal to the clamp and controlling the proximal hypertension. Perfusion of the lower part of the body can be achieved by the use of partial cardiopulmonary bypass and the use of different shunt techniques, this will reduce the incidence of postoperative renal dysfunction and paraplegia $[19,20]$. The advantages of distal perfusion strategies are, control of proximal hypertension, avoidance of renal and visceral ischemia, minimizing the degree of metabolic acidosis during clamping aorta, avoidance declamping shock, allowing rapid rewarming of the patients, an access for rapid fluid infusion, reduction the incidence of paraplegia and reduction of the incidence of disseminated coaulopathy. The disadvantages are, increased operative time, increased risk of air embolism, possibility of femoral, ventricular and atrial injury, increased blood loss due to the use of heparin and shunt dislodgement [21].

\section{Renal Protection During Surgery}

The incidence of acute postoperative renal insufficiency after DTA surgical intervention is $5-13 \%$. This is attributed to renal hypoper fusion during clamping aorta and reperfusion injury after removal of the clamp. Clamp time more than $30 \mathrm{~min}$ is associated with increased risk. Other predisposing factors include old age, pre-excising renal dysfunction, diabetes mellitus, hypovolemia and prolonged perioperative hypotension. Renal protective strategies include minimizing the aortic clamp time and maintenance of adequate intravascular volume and perfusion pressure. Distal perfusion techniques and the use of hypothermia have been shown to improve the postoperative renal function [22].

\section{Spinal Cord Protection During Surgery}

Acute paraplegia is a catastrophic complication of DTA clamping with an incidence of $5-30 \%$. The risk of paraplegia is increased in case of prolonged cross clamping of aorta ( $>30 \mathrm{~min}$ ) or injury of artery of Adamkiewcz. Other contributing risk factors are the occurrence of dissection of aorta, the aortic segment affected by the aneurysm and age [23]. Other predisposing factors include old age, pre-excising renal dysfunction, diabetes mellitus, hypovolemia and prolonged perioperative hypotension.

Cerebrospinal fluid (CSF) pressure is increased during aortic cross clamping with subsequent decrease in spinal cord perfusion pressure due to decreased mean arterial pressure and the elevated central venous pressure. Methylprednisolone $30 \mathrm{mg} / \mathrm{kg}$ and CSF drainage for at least for 48-72 hours after surgery to maintain CSF pressure at $10 \mathrm{~cm} \mathrm{H} 20$ with maintaining mean arterial pressure between 80 and $100 \mathrm{~mm} / \mathrm{Hg}$ can reduce the risk of spinal cord injury [24].

\section{Postoperative management}

a. Sedation: Sedation is maintained in the early postoperative period until hemodynamic and respiratory stabilization is achieved. Intermittent sedation holds should be used to allow assessment of neurological function [25].

b. Analgesia: Extensive surgical incision leads to significant pain that can impair weaning from sedation and artificial ventilation. A multimodal analgesic regimen is essential. Epidural analgesia has been advocated, but recently, bilateral paravertebral block with catheter insertion may represent a safe alternative for neuroaxial analgesia. PCA with opioids is another method for pain control. Adjuvant medications include paracetamol, ibuprofen, gabapentin and amirtyptaline [25].

c. Neurophysiological monitoring, ICP monitoring and CSF: Drainage should be continued for up to 72 hours after surgery. Monitoring of MEPs and SSEPs is continued whilst patients are sedated. Delayed paraplegia has a better prognosis than immediate, and can be reversed if recognized and treated promptly $[25,26]$.

d. Coagulation for drain removal: Platelet count>100/ML3; INR<1.3; normal APTT; delay removal 2-4 hours after last heparin dose; hold heparin 1 hour after catheter removal.

\section{Complications of major aortic surgery}

Whilst surgery for aortic aneurysm is potentially life-saving, there is a significant morbidity and mortality. Early complications include hypothermia, coagulopathy, delirium, cardiovascular instability, respiratory failure, metabolic disturbance, renal failure, and stroke. Because of the size of the surgical incision for DTA 
repair, and the potential for injury to the phrenic and recurrent laryngeal nerves, there is a significant risk of wound dehiscence and respiratory failure [27].

\section{Conclusion}

Anesthetic management for surgical repair of descending thoracic aortic aneurism remains a significant challenging condition. The main perioperative goals are prevention of renal, spinal cord, cardiac and cerebral dysfunction. Invasive monitoring, management of proximal hypertension and distal hypotension, the use of distal perfusion techniques, hypothermia and CSF drainage may reduce the incidence of renal, cardiac and spinal cord dysfunction.

\section{Acknowledgement}

\section{None}

\section{Conflict of Interest}

None.

\section{References}

1. Johnston KW, Rutherford RB, Tilson MD, Shah DM, Hollier L, et al. (1991) Suggested standards for reporting on arterial aneurysms. J Vasc Surg 13(3): 452-460.

2. Szilagyi DE, Smith RF, De Russo FJ, J P Elliott, F W Sherrin (1966) Contribution of abdominal aneurysmectomy to prolongation of life. Ann Surg 164(4): 678-699.

3. Crawford ES, Crawford JL, Safi HJ, Coselli JS, Hess KR (1986) Thoracoabdominal aortic aneurysms: preoperative and intraoperative factors determining immediate and long term results of operations in 605 patients. J Vasc Surg 3(3): 389-404.

4. Bickerstaff LK, Pairolero PC, Hollier LH, Melton LJ, Peenen HV, et al (1982) Thoracic aortic aneurysms: a population- based study. Surgery 92(6): 1103-1111.

5. Becker DA, Mc Garvey ML, Rojvirat C (2013) Predictors of outcome in patients with spinal cord ischaemia after open aortic repair. Neurocrit Care 18(1): 70-74.

6. Frederick JR, Woo YJ (2012) Thoracoabdominal aortic aneurysm. Ann Cardiothorac Surg 1(3): 277-285.

7. Hudson C, Coddens J, Swaminathan M (2010) Echocardiography for aortic surgery. In: Mathew JP, Swaminathan M, Ayoub CM (eds.) Clinical manual and review of trans-esophageal echocardiography $2^{\text {nd }}(e d n$.) McGraw Hill Education, USA

8. Madhusudan RP, Wei CL (2006) Descending thoracic aortic Aneurysms. Continuing Education in Anaesthesia, Critical Care \& Pain 6(2): 54-59.

9. Agarwal S, Kendall J, Quarterman C (2019) Perioperative management of thoracic and thoracoabdominal aneurysms. BJA Education 19(4): 119-125.

10. Simonetti G, Gandini G, Cornalba GP, Cassinis MC (2011) Diagnostic imaging of the aortic arch and thoraco-abdominal aorta. In: Chiesa $\mathrm{R}$, Melissano G, Zangrillo A (eds.) Thoraco Abdominal Aorta: Surgical and Anesthetic Management. Springer, USA, pp. 85-102.
11. Kihara C, Murata K, Wada Y, Hadano Y, Ohyama R, et al. (2009) Impact of intraoperative transesophageal echocardiography in cardiac and thoracic aortic surgery: experience with 1011 cases. J Cardiol 54(2): 282-290.

12. Kahn RA, Stone ME, Moskowitz DM (2007) Anesthetic Consideration for Descending Thoracic Aortic Aneurysm Repair. Semin Cardiothorac Vasc Anesth 11(3): 205-223.

13. Ouriel K, Shortell CK, Green RM, DeWeese JA (1993) Intraoperative auto transfusion in aortic surgery. J Vasc Surg 18(1): 16-22.

14. Hafez HM, Berwanger CS, Mc Coll A, Richmond W, Wolfe JHN, et al. (200) Myocardial injury in major aortic surgery. J Vasc Surg 31(4): 742-750.

15. Moskowitz DM, Kahn RA, Konstadt SN, Mitty H, Hollier LH, et al. (1999) Intraoperative Transoesophageal Echocardiography as an Adjuvant to Fluoroscopy during Endovascular Thoracic Aortic Repair. Eur J Vasc Endovasc Surg 17(1): 22-27.

16. Le Maire SA, Ochoa LN, Conklin LD, Widman RA, ClubbJr FJ, et al (2006) Transcutaneous near-infrared spectroscopy for detection of regional spinal ischemia during intercostal artery ligation: preliminary experimental results. J Thorac Cardiovasc Surg 132(5): 1150-1155.

17. Cruz KI, Le Maire SA, Weldon SA, Coselli JS (2012) Thoracoabdominal aortic aneurysm repair with a branched graft. Ann Cardiothor Surg 1(3): 381-393.

18. Gelman S (1995) The pathophysiology of aortic cross-clamping and unclamping. Anesthesiology 82(4): 1026-1060.

19. Svensson LG, Crawford ES (1992) Aortic dissection and aortic aneurysm surgery: clinical observations, experimental investigations and statistical analysis, Part I. Curr Probl Surg 29(11): 817-911.

20. Najafi H (1993) Descending aortic aneurysmectomy without adjuncts to avoid ischaemia. Ann Thorac Surg 55: 1042-1045.

21. Goel N, Jain D, Savlania A, Bansal A (2019) Thoracoabdominal Aortic Aneurysm Repair: What Should the Anaesthetist Know? Turk J Anaesthesiol Reanim 47(1): 1-11

22. Ling E, Arellano R (2000) Systematic overview of the evidence supporting the use of cerebrospinal fluid drainage in thoracoabdominal aneurysm surgery for the prevention of paraplegia. Anesthesiology 93(4): 1115-1122.

23. Safi HJ, Miller CC III, Huynh TT, Estrera AL, Porat E, et al. (2003) Distal aortic perfusion and cerebrospinal fluid drainage for thoracoabdominal and descending thoracic aortic repair: ten years of organ protection. Ann Surg 238(3): 372-380.

24. Drenger B, Parker SD, Frank SM, Beattie C (1997) Changesin cerebrospinal fluid pressure and lactate concentrations during thoracoabdominal aortic aneurysm surgery. Anesthesiology 86(1): 41-47.

25. Min HK, Sung K, Yang JH, Kim WS, Jun TG, et al. (2010) Can intraoperative motor- evoked potentials predict all the spinal cord ischemia during moderate hypothermic beating heart descending thoracic and thoracoabdominal aortic surgery? J Card Surg 25(5): 542-547.

26. Attaran S, Desmond M, Field M, Oo A (2010) Successful reversal of delayed paraplegia associated with chronic type an aortic dissection using a spinal drain. Interact Cardiovasc Thorac Surg 11(3): 374-379.

27. Le Maire SA, Price MD, Green S, Zarda S, Coselli JS (2012) Results of open thoracoabdominal aortic aneurysm repair. Ann Cardiothorac Surg 1(3): 286-292. 